\title{
Estimativa da produtividade da cana-de-açúcar para as principais regiões produtoras de Minas Gerais usando-se o método $Z^{2} E^{1}$
}

\author{
Robson A. Oliveira ${ }^{2}$ Roziane S. dos Santos ${ }^{2}$, Aristides Ribeiro², \\ Sérgio Zolnier ${ }^{2} \&$ Márcio H. P. Barbosa ${ }^{3}$
}

\begin{abstract}
RESU M O
O bjetivou-se, com este trabalho, parametrizar, calibrar e testar o método da Zona Agroecológica (ZAE) para as principais regiões produtoras de cana-de-açúcar do Estado de Minas Gerais. Foram utilizados dados meteorológicos do Instituto Nacional de Meteorologia e da Agência Nacional de Águas e dados de produtividade do Programa de M elhoramento $G$ enético da Cana-de-Açúcar. 0 método ZAE apresentou bom ajuste, com $\mathrm{R}^{2}=0,89 ; \mathrm{RMSE}=8,4 \mathrm{tha}^{-1} ; \mathrm{MAE}=6,7 \mathrm{tha}^{-1} ; \mathrm{MBE}=5,9 \mathrm{t}^{-1} \mathrm{e}$ índice de Wilmont (d) $=0,95$. $\mathrm{N}$ a validação realizada mediante a utilização de uma série temporal de dados de produtividade do Instituto Brasileiro de Geografia e Estatística das safras de 1989/90 a 2007/08, referente à região do Triângulo Mineiro, constatou-se um erro sistemático com tendência de superestimativa da produtividade de aproximadamente $37 \mathrm{t} \mathrm{ha}^{-1}$. Corrigindo-se a tendência de superestimativa o método ZAE apresentou ajuste satisfatório $\left(\mathrm{R}^{2}=0,55 ; \mathrm{RMSE}=4,9 \mathrm{t} \mathrm{ha}^{-1} ; \mathrm{MAE}=4,0\right.$ t ha-1; $\mathrm{MBE}=0,0 \mathrm{t} \mathrm{ha}^{-1} ;$ índice $\mathrm{d}=0,80$ ). Assim, este modelo se apresentou como importante ferramenta para estimar a produtividade da cana-deaçúcar em macroescala no Estado, podendo auxiliar no planejamento da expansão otimizada da atividade na região.
\end{abstract}

Palavras-chave: balanço hídrico, clima, modelo agrometeorológico, zona agroecológica

\section{Yield estimate of sugarcane in main producing regions of Minas Gerais using the AEZ method}

\begin{abstract}
A B ST RACT
The objective of this study was to parameterize, calibrate and test the Agroecological Zone (AEZ) method for estimating the yield of sugarcane in the major producing areas of sugarcane in the State of Minas Gerais. M eteorological data from the Instituto Nacional de Meteorologia and the Agência Nacional de Águas, as well as productivity data from Programa de M elhoramento G enético da Cana-de-Açúcar were used. The AEZ method showed good agreement, $R^{2}=0.89, \mathrm{RMSE}=8.4 \mathrm{tha}^{-1} ; \mathrm{MAE}=6.7 \mathrm{tha}^{-1}, \mathrm{MBE}=$ 5.9 t ha $^{-1}$ and Willmott's coefficient $(\mathrm{d})=0.95$. For validation, a series of crop productivity data from the Instituto Brasileiro de Geografia e Estatística from 1989/90 to 2007/08 was used for the Triângulo M ineiro region. A systematic error with a tendency to overestimate the productivity of about $37 \mathrm{th} \mathrm{a}^{-1} \mathrm{w}$ as found. After correcting the tendency of overestimating, the AEZ method showed satisfactory agreement $\left(R^{2}=\right.$ $0.55, \mathrm{RMSE}=4.9 \mathrm{t} \mathrm{ha}^{-1} ; \mathrm{MAE}=4.0 \mathrm{t} \mathrm{ha}^{-1}, \mathrm{MBE}=0.0 \mathrm{t} \mathrm{ha}^{-1}$ and $\mathrm{d}=0.80$ ). Therefore, the model has revealed to be an important tool for estimating the yield of sugarcane in a large scale in the state, which could be useful to plan the expansion of optimal activity in the region.
\end{abstract}

Key words: water balance, climate, agrometeorological model, agroecological zone 


\section{INTRODUÇÃO}

As áreas cultivadas com cana-de-açúcar têm apresentado uma rápida expansão no Estado de Minas Gerais, com o planejamento e implantação de usinas sucroalcooleiras no interior de Minas Gerais, principalmente nas regiões Noroeste de Minas, Central Mineira, Sul/Suldoeste e Triângulo Mineiro. Atualmente, existem 43 usinas de açúcar e etanol em produção no estado, das quais 23 localizadas no Triângulo, representando 69\% do total da produção mineira (IOMG, 2010).

Segundo Doorenbos \& Kassam (1979) a temperatura é um dos elementos meteorológicos mais importantes na produção da cana-de-açúcar, cuja diminuição tem importância fundamental no processo de maturação. Temperaturas amenas são responsáveis pela redução do ritmo vegetativo para o acúmulo de sacarose nos colmos (Scarpari \& Beauclair, 2009). Outro elemento relevante que deve ser destacado é a disponibilidade de água visto que nem sempre as chuvas atendem à real necessidade hídrica das plantas (Dantas Neto et al., 2006), uma vez que toda cultura de sequeiro está à mercê da quantidade e da distribuição das chuvas. O déficit hídrico, por sua vez, afeta vários aspectos do crescimento vegetal destacando-se a redução do tamanho das plantas, de sua área foliar e da produtividade da cultura (Farias et al., 2008). A produtividade da cana-de-açúcar é altamente dependente das interações entre suas fases fenológicas e as variações inter e intranuais do clima. Portanto, a utilização de um modelo de estimativa de produtividade exerce importante papel econômico, sendo uma ferramenta eficaz para estimativa de produção em diferentes condições climáticas (Silva et al., 2009).

Os modelos de estimativa da produtividade baseados em fatores climáticos permitem acompanhar os efeitos do clima ao longo do ciclo da cultura e relacioná-los com a produtividade final, tornando-se possível avaliar as restrições ao desenvolvimento agrícola e obter estratégias para o incremento da produção (Tejera et al., 2007; Gilbert et al., 2006; Singels et al., 2005a; Inman-Bamber et al., 2002). Utilizando-se a previsão climática com 2 a 3 meses de antecedência, pode-se estimar quebras futuras na safra corrente devido a um eventual período menos chuvoso ou veranico, bem como estimar a produtividade máxima possível para um período com a quantidade ideal de chuvas, servindo como ferramenta de manejo. Os modelos também podem ser utilizados como ferramenta de planejamento agrícola, estabelecendo uma estimativa de produtividade potencial e analisando-se o efeito dos extremos climáticos na produtividade futura, bem como no estudo das variações interanual e inter-regional.

Desta forma, a aplicação dos modelos de simulação na agricultura possibilita uma economia de tempo, trabalho e quantidade de recursos, constituindo uma ferramenta que pode ser um importante subsídio para a definição de políticas públicas de tomada de decisão. Ainda mais, os resultados das simulações proporcionam conhecimentos que possibilitam recomendar práticas para redução dos riscos ambientais e dos custos de produção, resultando em maior sustentabilidade do planejamento agrícola (Gouvêa et al., 2009). Everingham et al. (2009) enfatizam que as previsões de produção são úteis para o sucesso de qualquer indústria agrícola que planeja ou vende a produção antes da colheita.

Dentre os modelos de simulação de produtividade disponíveis se destaca o Método da Zona Agroecológica (ZAE), desenvolvido por Kassam (Fischer et al., 2002; Doorenbos \& Kassam, 1979), sendo uma forma muito precisa para a estimativa da produtividade potencial das culturas em função do clima (Tubiello et al., 2007). Além disso, esta produtividade potencial pode ser penalizada pelo déficit hídrico, de vez que este modelo é capaz de estimar a produtividade real com alta precisão (Gouvêa et al., 2009; Marin et al., 2008). O método ZAE é de fácil entendimento e aplicação em razão da baixa exigência de dados de entrada (dados meteorológicos e da cultura), apresentando resultados bastante consistentes que se aproximam da realidade, rápidos e a um baixo custo. Entretanto, o modelo apresenta algumas limitações pois não são consideradas a ocorrência de pragas e doenças nem a fertilidade do solo. Apesar de suas limitações, pela sua simplicidade e bom desempenho, o método ZAE tem sido bastante utilizado. Bonnecarrère et al. (2007) aplicaram o método ZAE para estimar a produtividade potencial e deplecionada para a cultura de milho no Rio Grande do Sul e obtiveram bons resultados. Gouvêa et al. (2009) utilizaram o método ZAE para determinar a produtividade potencial e os riscos climáticos associados à produção da cana-de-açúcar diante do cenário de mudanças climáticas na região de Piracicaba, SP.

Com o exposto e se considerando a relevância da cultura da cana-de-açúcar na agricultura do Estado de Minas Gerais, torna-se necessário a utilização de um modelo simplificado de predição de produtividade que seja adaptado à realidade deste Estado. Assim, este estudo teve por objetivo parametrizar e calibrar o método da ZAE para a estimativa da produtividade da cana-de-açúcar limitada pela disponibilidade hídrica (em condições de sequeiro), de modo a torná-lo aplicável à previsão de safra a partir de dados meteorológicos e da cultura para as principais regiões produtoras do Estado, testando-o para a região do Triângulo Mineiro.

\section{Material e MÉTOdos}

A área de estudo foi o Estado de Minas Gerais, sendo a região do Triângulo Mineiro usada para testar o método ZAE. Esta região foi escolhida devido ao seu maior potencial de expansão da cultura da cana-de-açúcar. O Estado de Minas Gerais está situado na região Sudeste do Brasil, entre os paralelos $14^{\circ} 13^{\prime} 57^{\prime \prime}$ e $22^{\circ} 55^{\prime} 22^{\prime}$ ' S e os meridianos de $39^{\circ} 51^{\prime}$ 23 " e $51^{\circ} 02$ ' $45^{\prime \prime}$ W. O Triângulo Mineiro é uma das dez regiões de planejamento do Estado.

\section{Método da zona agroecológica (ZAE)}

O método ZAE calcula a produção potencial de biomassa em função da temperatura do ar, da irradiância solar e da duração do ciclo da cultura, além de fatores genético; em seguida, a produtividade potencial é penalizada utilizando-se o coeficiente de sensibilidade da produtividade ao déficit de umidade no solo $\left(\mathrm{K}_{\mathrm{y}}\right)$. A produtividade potencial da cultura da cana-de- 
açúcar $\left(\mathrm{PP}_{\text {cana }}\right)$ representa o nível de referência de produtividade atingível com alto padrão de manejo da cultura e água, em que não há limitações de clima, água e nutrientes e sem ataque de pragas e doenças (Doorenbos \& Kassam, 1979). O método da ZAE determina $\mathrm{PP}_{\text {cana }}\left(\mathrm{kg} \mathrm{ha}^{-1}\right)$ por meio da Eq.1:

$$
\begin{aligned}
\mathrm{PP}_{\text {cana }} & =\mathrm{C}_{\text {iaf }} \cdot \mathrm{C}_{\mathrm{r}} \cdot \mathrm{C}_{\mathrm{col}} \cdot \mathrm{C}_{\mathrm{um}} \cdot \mathrm{ND} \cdot\left[\mathrm{F} \cdot\left(\mathrm{a}+\mathrm{b} \cdot \mathrm{PPB}_{\mathrm{p}}\right) \cdot \mathrm{PB}_{\mathrm{n}}+\right. \\
& \left.+(1-\mathrm{F}) \cdot\left(\mathrm{c}+\mathrm{d} \cdot \mathrm{PPB}_{\mathrm{p}}\right) \cdot \mathrm{PB}_{\mathrm{c}}\right]
\end{aligned}
$$

na qual $\operatorname{PPB}_{\mathrm{P}}\left(\mathrm{kg} \mathrm{MS} \mathrm{ha}^{-1} \mathrm{~d}^{-1}\right)$ é a taxa de produção bruta de matéria seca da cultura-padrão e os demais fatores adimensionais são descritos a seguir.

Para determinação da $\mathrm{PP}_{\text {cana }}$, se adotaram os coeficientes de correção do índice de área foliar, respiração e índice de colheita, abaixo descritos conforme estabelecidos para a cultura da canade-açúcar, por Doorenbos \& Kassam (1979) no documento FAO-33. O coeficiente $\left(\mathrm{C}_{\text {iaf }}\right)$ visa ajustar o índice de área foliar da cultura da cana-de-açúcar a uma condição mais próxima da realidade.

O método ZAE pressupõe uma relação direta entre a temperatura e a respiração; portanto, é necessário considerar um coeficiente de correção para a respiração $\left(\mathrm{C}_{\mathrm{r}}\right)$ na estimativa da produtividade máxima. Conforme especificado por Doorenbos \& Kassam (1979), adotou-se $\mathrm{C}_{\mathrm{r}}$ como sendo igual a 0,5 para temperaturas médias e" $20{ }^{\circ} \mathrm{C}$ e igual a 0,6 para temperaturas médias $<20^{\circ} \mathrm{C}$. O índice de colheita $(\mathrm{Ccol})$ é a relação entre a matéria seca economicamente rentável e a massa seca total produzida. De acordo com Doorenbos \& Kassam (1979), o valor 0,80 pode ser adotado. O coeficiente (Cum) é utilizado para inserir a umidade residual na matéria seca da parte rentável que, normalmente, fica retida (Doorenbos \& Kassam 1979). ND é a duração do ciclo de crescimento (dias). Os valores de a, b, c, d são iguais a 0,$8 ; 0,01 ; 0,5$ e 0,025 respectivamente, para PPBp e" $20 \mathrm{~kg} \mathrm{ha}^{-1} \mathrm{~d}^{-1}$ e iguais a 0,5 ; 0,025; 0 e 0,05; respectivamente, para PPBp $<20 \mathrm{~kg} \mathrm{ha}^{-1} \mathrm{~d}^{-1}$ (Fischer et al., 2002; Doorenbos \& Kassam, 1979).

A fração do dia com nebulosidade (F) foi calculada durante todo o ciclo da cultura pela Eq. 2, utilizado-se um valor médio como variável de entrada na Eq. 1.

$$
\mathrm{F}=\left(\frac{\mathrm{R}_{\mathrm{so}} \cdot 0,5 \cdot \mathrm{R}_{\mathrm{s}}}{0,8 \cdot \mathrm{R}_{\mathrm{so}}}\right)
$$

em que:

$\mathrm{R}_{\mathrm{so}}$ - irradiância solar na superfície para dias claros, $\mathrm{MJ} \mathrm{m}^{-2} \mathrm{~d}^{-1}$

$\mathrm{R}_{\mathrm{s}}^{\mathrm{so}}$ - irradiância solar global à superfície, $\mathrm{MJ} \mathrm{m}^{-2} \mathrm{~d}^{-1}$

Devido às falhas nos dados de insolação e à indisponibilidade de dados medidos de irradiância solar global incidente na superfície terrestre $\left(R_{s}\right)$ nas estações meteorológicas de superfície convencional do INMET, adotouse a metodologia proposta por Bristow \& Campbell (1984) para estimativa da irradiância solar global, conforme a Eq. 3:

$$
\mathrm{R}_{\mathrm{s}}=\mathrm{R}_{\mathrm{o}} \cdot \mathrm{A} \cdot\left[1-\mathrm{e}^{\left(-\mathrm{B} \cdot \Delta \mathrm{T}^{\mathrm{C}}\right)}\right]
$$

em que:

$\mathrm{R}_{\mathrm{o}}$ - irradiância solar no topo da atmosfera, $\mathrm{MJ} \mathrm{m}^{-2} \mathrm{~d}^{-1}$

$\Delta \mathrm{T}$ - variação diária das temperaturas máxima e mínima do ar, ${ }^{\circ} \mathrm{C}$, sendo A, B e C constantes empíricas, com valores de 0,7; 0,007 e 2,4, respectivamente. O parâmetro "A" representa a irradiância máxima em um dia de céu claro e os parâmetros "B" e "C" são os controladores da variação de "A", caso ocorra aumento na diferença de temperatura. A irradiância solar no topo da atmosfera $\left(\mathrm{R}_{0}\right)$ e a irradiância solar na superfície para dias claros $\left(\mathrm{R}_{\mathrm{so}}\right)$ foram obtidas conforme a metodologia apresentada no documento FAO-56 (Allen et al., 1998).

A variação da temperatura $(\Delta \mathrm{T})$ foi calculada pela Eq. 4 :

$$
\Delta \mathrm{T}=\mathrm{t}_{\mathrm{x}}-\left(\frac{\mathrm{t}_{\mathrm{n}}+\mathrm{t}_{\mathrm{n}+1}}{2}\right)
$$

em que:

$$
\begin{aligned}
& t_{x} \quad \text { - temperatura máxima do dia, }{ }^{\circ} \mathrm{C} \\
& t_{n} \text { - temperatura mínima do dia, }{ }^{\circ} \mathrm{C} \\
& t_{n+1}-\text { temperatura mínima do dia posterior, }{ }^{\circ} \mathrm{C}
\end{aligned}
$$

Como durante um dia ocorrem, com bastante frequência, períodos de nebulosidade, a $\mathrm{PP}_{\text {cana }}$ é composta de um componente relativo ao período nublado $\mathrm{PB}_{\mathrm{n}}\left(\mathrm{kg} \mathrm{MS} \mathrm{ha}^{-1} \mathrm{~d}^{-1}\right) \mathrm{e}$ outro ao período de céu claro $\mathrm{PB}_{\mathrm{c}}\left(\mathrm{kg} \mathrm{MS} \mathrm{ha}^{-1} \mathrm{~d}^{-1}\right)$. Deste modo, a $\mathrm{PB}_{\mathrm{c}}$ e a $\mathrm{PB}_{\mathrm{n}}$ são calculadas diariamente, durante todo o ciclo da cultura, pelas Eqs. 5 e 6, propostas por Leal (2000).

$$
\begin{aligned}
& \mathrm{PB}_{\mathrm{n}}=31,653+0,5477 \cdot \mathrm{R}_{\mathrm{so}} \\
& \mathrm{PB}_{\mathrm{c}}=104,66+0,9061 \cdot \mathrm{R}_{\mathrm{so}}
\end{aligned}
$$

A taxa de produção bruta de matéria seca da cultura-padrão $\left(\mathrm{PPB}_{\mathrm{p}}\right.$ ) é considerada como tendo o valor de $20 \mathrm{~kg} \mathrm{ha}^{-1} \mathrm{~d}^{-1}$; entretanto, a produção da matéria seca depende da cultura e da temperatura do ar $\left(\mathrm{t},{ }^{\circ} \mathrm{C}\right)$. Assim, os valores da $\mathrm{PPB}_{\mathrm{P}}$ para a cultura da cana-de-açúcar foram equacionados em função da temperatura do ar para o intervalo $5^{\circ}$ a $45^{\circ} \mathrm{C}$, conforme valores propostos por Fischer et al. (2002) e Doorenbos \& Kassam (1979), demonstrados nas equações abaixo:

$$
\operatorname{Para}^{\circ} \leq \mathrm{t} \leq 10^{\circ} ; \quad \mathrm{PPB}_{\mathrm{p}}=0
$$

$$
\operatorname{Para} 10^{\circ}<\mathrm{t} \leq 15^{\circ} ; \quad \operatorname{PPB}_{\mathrm{p}}=\mathrm{t}-10
$$

Para $15^{\circ}<\mathrm{t} \leq 25^{\circ} ; \quad \mathrm{PPB}_{\mathrm{p}}=-0,397 \cdot \mathrm{t}^{2}+21,916 \cdot \mathrm{t}-236,52, \mathrm{R}^{2}=0,99$

$$
\text { Para } 25^{\circ}<\mathrm{t} \leq 35^{\circ} ; \quad \mathrm{PPB}_{\mathrm{p}}=65
$$

Para $35^{\circ}<\mathrm{t} \leq 45^{\circ} ; \quad \mathrm{PPB}_{\mathrm{p}}=-0,350 \cdot \mathrm{t}^{2}+21,972 \cdot \mathrm{t}-276,40, \mathrm{R}^{2}=0,99$

\section{Evapotranspiração da cultura (ETc cana $)$}

No cálculo da produtividade limitada pela disponibilidade hídrica constata-se que a evapotranspiração máxima da cultura 
$\left(\mathrm{ETc}_{\text {cana }}\right)$ é relacionada com a evapotran spiração de referência $\left(\mathrm{ET}_{\mathrm{o}}\right)$ por meio de um coeficiente de cultura $\left(\mathrm{K}_{\mathrm{c}}\right)$, como apresentado na Eq. 12:

$$
\mathrm{ETc}_{\text {cana }}=\mathrm{K}_{\mathrm{c}} \cdot \mathrm{ET}_{\mathrm{o}}
$$

A evapotranspiração de referência foi calculada de acordo com a Equação de Penman-Moneith/FAO-56 (Allen et al., 1998). Foram adotados 0,$4 ; 1,25$ e 0,75 , como valores de coeficiente de cultura para os estádios inicial; reprodutivo e de maturação, respectivamente, conforme proposto por Doorenbos \& Kassam (1979).

A duração (em dias) do primeiro estádio de desenvolvimento da cultura (estádio inicial) foi definida como de 35 dias para a cana planta e 25 dias para a cana-soca. Os demais estádios (vegetativo, reprodutivo e de maturação) foram estabelecidos com base no método dos graus-dia acumulados (Liu et al., 1998; Liu \& Bull, 2001; Singels et al., 2005b), através dos valores encontrados por Almeida et al. (2008). Desta forma foram uitilizados, para a fase de estabelecimento da cultura, 750 Grausdia Acumulados (GDA, ${ }^{\circ} \mathrm{C} \mathrm{d}^{-1}$ ) para a cana-planta e 600 GDA para a cana-soca; já para o estádio reprodutivo se adotaram 750 e 1500 GDA para a cana-planta e 600 e 950 GDA para a cana-soca, respectivamente.

\section{Balanço hídrico}

Por ser o hídrico um dos fatores que mais atuam na produtividade das culturas, faz-se necessário incluir esta variável nos modelos de estimativa da produtividade associando, assim, os elementos chuva e evapotranspiração sobre a produtividade, para fases fenológicas da cultura (Delgado-Rojas \& Barbieri, 1999).

Para a estimativa do balanço hídrico sequencial diário adotou-se o procedimento proposto por Thornthwaite \& Mather (1955), utilizando-se $78 \mathrm{~mm}$ para o valor da capacidade de água disponível no solo (CAD) (Doorenbos \& Kassam, 1979).

Quando as necessidades hídricas da cultura são atendidas plenamente, a evapotranspiração real $\left(E_{\mathrm{r}}\right)$ é igual à evapotranspiração da cultura $\left(\mathrm{ET}_{\mathrm{c}}\right)$. Todavia, quando o suprimento é insuficiente tem-se que $\mathrm{ET}_{\mathrm{r}}<\mathrm{ET}_{\mathrm{c}}$ e a perda de produtividade é proporcional ao estresse hídrico sofrido pela cultura. A PR ${ }_{\text {cana }}$ (Produtividade da cana-de-açúcar limitada pela disponibilidade hídrica ao longo do ciclo) obtida pelo método ZAE, é resultante da $\mathrm{PP}_{\text {cana }}$ penalizada pelo estresse hídrico e obtida pelo fator (1 - ${ }_{\text {ETr }}{ }_{\mathrm{ETc}}{ }^{\text {cana }}$, em função da sua sensibilidade ao déficit hídrico $\left(\mathrm{K}_{\mathrm{y}}\right)$, utilizando-se a Eq. 13.

$$
\mathrm{PR}_{\text {cana }}=\mathrm{PP}_{\text {cana }} \times\left[1-\mathrm{k}_{\mathrm{y}} \cdot\left(1-\frac{\mathrm{ETr}_{\text {cana }}}{\mathrm{ETc}_{\text {cana }}}\right)\right]
$$

em que:

$\mathrm{k}_{\mathrm{y}}$ - 1,20 para todo o ciclo da cultura da cana-de-açúcar (Doorenbos \& Kassam, 1979).

\section{Base de dados}

Visando à simulação de produtividade da cana-de-açúcar pelo método ZAE, foram utilizados dados meteorológicos diários de temperatura máxima do ar, temperatura mínima do ar, umidade relativa média do ar, velocidade média do vento e precipitação, no período de 2001 a 2008, obtidos das estações climatológicas de superfície convencional do Instituto Nacional de Meteorologia (INMET).

Com o intuito de melhor representar a precipitação foram utilizados, também, dados das estações pluviométricas da Agência Nacional de Águas (ANA), no período de 2001 a 2008, localizadas próximo às empresas produtoras de cana-de-açúcar.

Utilizaram-se, para calibração e parametrização do método ZAE, dados de produtividade em toneladas de colmos por hectare, provenientes do Programa de Melhoramento Genético da Cana-de-açúcar da Universidade Federal de Viçosa (PMGCAUFV), cujo objetivo principal é desenvolver variedades de canade-açúcar por meio da cooperação técnica firmada com usinas e destilarias produtoras de açúcar, etanol e energia, no estado de Minas Gerais. A Tabela 1 apresenta as usinas e destilarias utilizadas neste estudo, as respectivas estações do INMET e o código das estações da ANA adotadas em cada caso. A Figura 1 apresenta a localização geográfica das usinas e destilarias utilizadas.

Tabela 1. U sinas (U ) e destilarias (D), estações do IN M ET da ANA utilizadas

\begin{tabular}{lllc}
\hline \multirow{2}{*}{ Nome } & \multicolumn{1}{c}{ Cidade } & \multicolumn{2}{c}{ Estação } \\
\cline { 3 - 4 } D. Agropeu & Pompéu & Pompéu & ANA \\
Canacampo & Campo Florido & Uberaba & - \\
D. R. Cachimbo & João Pinheiro & J oão Pinheiro & - \\
D. WD & João Pinheiro & J oão Pinheiro & - \\
U. Passos & Passos & Uberaba & 2046011 \\
U. Alvorada & Araporã & Capinópolis & 1849006 \\
U. Iturama & Iturama & Ituiutaba & 1950012 \\
U. Jatiboca & Urucânia & Viçosa & 2042011 \\
U. Luciania & Lagoa da Prata & Pompéu & 2045011 \\
U. Santo Angelo & Pirajuba & Uberaba & 1948010 \\
U. Trialcool & Canápolis & Ituiutaba & - \\
U. Volta Grande & C. das Alagoas & Uberaba & - \\
\hline
\end{tabular}

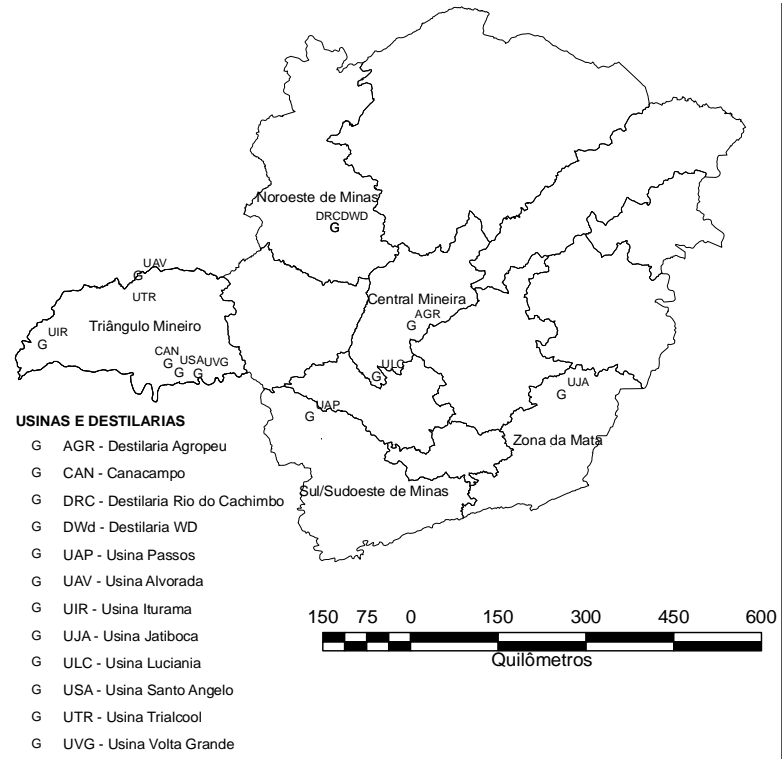

Figura 1. Localização das U sinas e Destilarias do PMGCA utilizadas neste estudo 
Para a escolha dos experimentos utilizados neste estudo fez-se o controle de qualidade com o objetivo de identificar valores anômalos na base de dados do PMGCA-UFV, os quais foram excluídos das análises dos experimentos com valores muito altos e muito baixos de produtividade. Foram utilizados os 30 experimentos que melhor representaram a produtividade média da variedade RB835486, dentre eles 15 experimentos para o ciclo da cana-planta e 15 para o ciclo da primeira cana-soca, listados nas Tabelas 2 e 3 . A variedade RB835486 foi escolhida por ter apresentado uso generalizado nos experimentos utilizados, permitindo comparações.

Tabela 2. Experimentos utilizados para o ciclo da canaplanta, com as respectivas datas de plantio e corte e duração do ciclo

\begin{tabular}{ccccc}
\hline Empresa & Exp. & Plantio & 10 corte & Ciclo* \\
AGR & 1 & $09 / 03 / 2005$ & $09 / 08 / 2006$ & 518 \\
AGR & 2 & $06 / 04 / 2004$ & $27 / 07 / 2005$ & 477 \\
CAN & 3 & $06 / 04 / 2004$ & $29 / 06 / 2005$ & 449 \\
DRC & 4 & $23 / 04 / 2003$ & $11 / 05 / 2004$ & 384 \\
DWD & 5 & $15 / 03 / 2006$ & $17 / 07 / 2007$ & 489 \\
UAP & 6 & $10 / 03 / 2004$ & $13 / 07 / 2005$ & 490 \\
UAP & 7 & $29 / 08 / 2006$ & $29 / 08 / 2007$ & 365 \\
UAV & 8 & $07 / 04 / 2004$ & $19 / 07 / 2005$ & 468 \\
UJA & 9 & $30 / 09 / 2004$ & $04 / 10 / 2005$ & 369 \\
USA & 10 & $29 / 03 / 2004$ & $30 / 06 / 2005$ & 458 \\
USA & 11 & $03 / 05 / 2007$ & $20 / 08 / 2008$ & 475 \\
UTR & 12 & $25 / 03 / 2004$ & $15 / 07 / 2005$ & 477 \\
UTR & 13 & $18 / 02 / 2006$ & $11 / 04 / 2007$ & 417 \\
UTR & 14 & $03 / 03 / 2006$ & $16 / 04 / 2007$ & 409 \\
UTR & 15 & $13 / 04 / 2006$ & $08 / 08 / 2007$ & 482 \\
\hline
\end{tabular}

Tabela 3. Experimentos utilizados para o ciclo da primeira cana-soca, com as respectivas datas dos cortese duração do ciclo

\begin{tabular}{cccccc}
\hline Empresa & Exp. & $\mathbf{1 0}$ corte & $\mathbf{2 0}$ corte & Ciclo* & Maturação** \\
AGR & 16 & $09 / 08 / 2006$ & $18 / 07 / 2007$ & 343 & média \\
AGR & 17 & $27 / 07 / 2005$ & $26 / 06 / 2006$ & 334 & precoce \\
CAN & 18 & $29 / 06 / 2005$ & $01 / 08 / 2006$ & 398 & média \\
DRC & 19 & $08 / 08 / 2001$ & $07 / 08 / 2002$ & 364 & média \\
DWD & 20 & $15 / 08 / 2005$ & $22 / 08 / 2006$ & 372 & média \\
DWD & 21 & $22 / 08 / 2006$ & $17 / 07 / 2007$ & 329 & média \\
DWD & 22 & $17 / 07 / 2007$ & $16 / 07 / 2008$ & 365 & média \\
UIR & 23 & $22 / 08 / 2003$ & $16 / 07 / 2004$ & 329 & média \\
UIR & 24 & $18 / 07 / 2004$ & $09 / 08 / 2005$ & 387 & média \\
ULC & 25 & $13 / 07 / 2004$ & $02 / 08 / 2005$ & 385 & média \\
USA & 26 & $30 / 06 / 2005$ & $27 / 07 / 2006$ & 392 & média \\
UTR & 27 & $15 / 07 / 2005$ & $07 / 07 / 2006$ & 357 & média \\
UTR & 28 & $13 / 04 / 2007$ & $29 / 05 / 2008$ & 412 & precoce \\
UTR & 29 & $11 / 04 / 2007$ & $18 / 03 / 2008$ & 342 & precoce \\
UVG & 30 & $30 / 04 / 2004$ & $20 / 04 / 2005$ & 355 & precoce \\
\hline
\end{tabular}

* Ciclo em dias ** Maturação: precoce, média e tardia

A validação do método ZAE foi feita para a região do Triângulo Mineiro, através de uma série temporal de dados de produtividade observada, obtida junto ao Instituto Brasileiro de Geografia e Estatística (IBGE), disponível no Sistema de Recuperação Automática (SIDRA) para as safras de 1989/90 a 2007/08. três diferentes condições de manejo para a cana-soca em cada safra foram simuladas, todas com duração de 365 dias, a partir de dados meteorológicos das estações meteorológicas de superfície convencional do INMET localizadas em Uberaba, Capinópolis e Ituiutaba; a primeira simulação, compreendida entre maio e abril do ano seguinte, buscou representar uma soqueira de corte precoce; a segunda, entre agosto de um ano até julho do ano seguinte, representando as soqueiras de corte no meio da safra e a terceira, entre novembro e outubro, representando as áreas de colheita tardia. Para cada safra o valor final de produtividade foi considerado como sendo a média aritmética dessas três simulações das três estações utilizadas.

Verificou-se o desempenho do método ZAE por meio dos seguintes indicadores estatísticos: coeficiente de determinação $\left(\mathrm{R}^{2}\right)$, raiz do erro quadrático médio (RMSE, sigla em inglês), o erro absoluto médio (MAE, sigla em inglês), o viés médio(MBE, sigla em inglês) e o índice de concordância de Willmott (d) (Willmott, 1982).

\section{RESULTADOS E DISCUSSÃO}

\section{Parametrização e calibração do método ZAE}

Na Figura 2 são apresentadas as relações entre os valores de produtividade simulados pelo método ZAE e observados em campo (PMGCA-UFV) para a variedade RB835486, tanto para o ciclo da cana-planta como para o primeiro ciclo da canasoca.

Verifica-se que a produtividade média observada para o ciclo da cana-planta foi de $107,4 \mathrm{t} \mathrm{ha}^{-1}$ e a produtividade média estimada pelo método ZAE foi de 108,3 $\mathrm{t} \mathrm{ha}^{-1}$ (Figura 2A). Na análise de todo o conjunto de dados observados e estimados observa-se que o método ZAE apresentou bom ajuste aos dados observados, não evidenciando tendência de superestimativa ou subestimativa, com boa exatidão $(\beta 1=0,80)$ e boa precisão $\left(\mathrm{R}^{2}=0,77\right)$, explicando $77 \%$ da variabilidade dos dados observados em experimentos de campo. Nesta análise o intercepto da equação de regressão foi desprezado em virtude de não ser estatisticamente significativo a nível de $5 \%$. Analisando o desempenho estatístico do método ZAE para estimativa da produtividade limitada pela disponibilidade hídrica ao longo do ciclo de cana-planta, observam-se baixos valores dos erros RMSE e MAE (7,1 t ha ${ }^{-1}$ e 5,6 t ha ${ }^{-1}$, respectivamente), indicando que o método apresenta bom ajuste, fato passível de ser confirmado com o valor do $\mathrm{MBE}=1,0 \mathrm{t} \mathrm{ha}^{-1}$, ou seja, em média, o método é bem coerente com os dados experimentais para o ciclo da cana-planta, sinalizando apenas uma pequena superestimação de 1,0 t ha-1. Além disso, o alto valor do índice de concordância de Willmott $(\mathrm{d}=0,94)$ indica boa concordância dos dados estimados e observados, corroborando com a eficácia do método (Figura 2A).

A média da produtividade observada para o ciclo da primeira cana-soca foi de 101,5 $\mathrm{t} \mathrm{ha}^{-1}$ e a produtividade média estimada pelo método ZAE foi de 104,4 $\mathrm{t} \mathrm{ha}^{-1}$ (Figura 2B). Nota-se que o método ZAE apresentou um ajuste satisfatório para o primeiro ciclo de cana-soca, explicando $89 \%$ da variabilidade dos dados observados em campo. A exatidão do método para a cana-soca, dada pelo coeficiente angular da regressão $(\beta 1=0,90)$ e a precisão, dada pelo coeficiente de determinação $\left(r^{2}=0,89\right)$ foram superiores às obtidas para a cana-planta. Novamente o 
A.

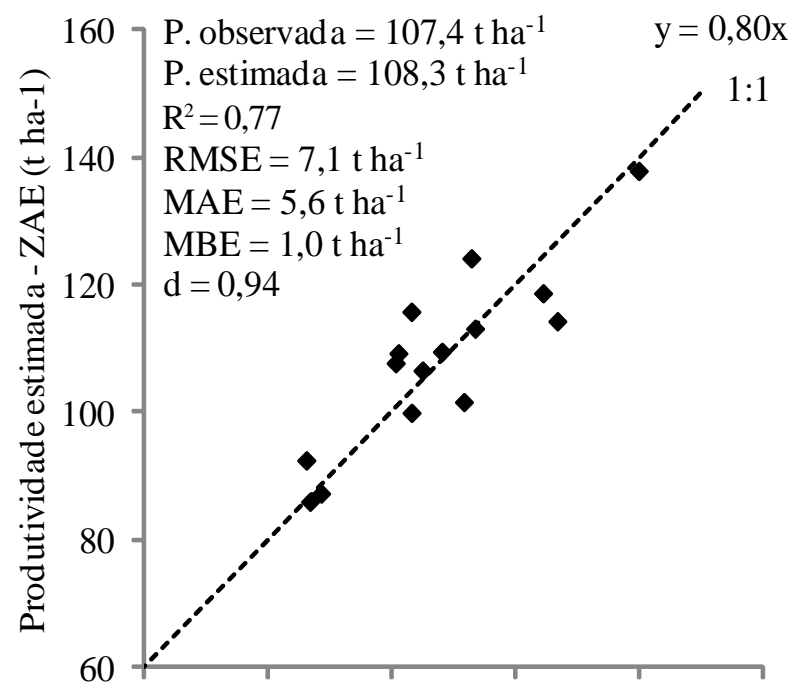

B.

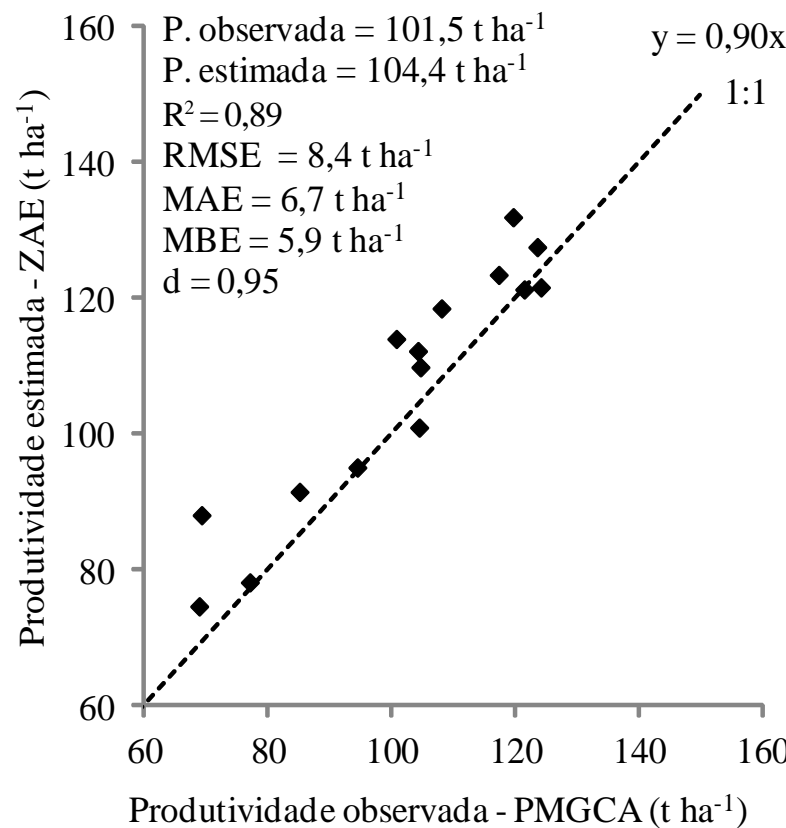

Figura 2. Relação entre a produtividade limitada pela disponibilidade hídrica estimada pelo método ZAE e a produtividade observada em campo nos experimentos do PM GCA-UFV para o ciclo da cana-planta (A) e primeiro ciclo da cana-soca (B)

intercepto da equação da equação de regressão foi desprezado por não ser estatisticamente significativo a nível de 5\%. Semelhante aos resultados encontrados para o ciclo da canaplanta obtiveram-se baixos valores dos erros RMSE e MAE $\left(8,4 \mathrm{tha}^{-1} \mathrm{e} 6,7 \mathrm{tha}^{-1}\right.$, respectivamente) para o primeiro ciclo de cana-soca. Contudo, o MBE $=5,9 \mathrm{t} \mathrm{ha}^{-1}$ foi um pouco elevado indicando uma superestimativa (Figura 2B). Esta tendência era prevista para ambos os casos e pode ser associada ao fato de que se assumiu apenas a água como fator limitante da produtividade desconsiderando, portanto, outros fatores imprescindíveis na definição da produtividade da cultura, como doenças, pragas, carências nutricionais e o manejo inadequado (Doorenbos \& Kassam, 1979). Apesar disto e mesmo com essa pequena tendência do método ZAE em superestimar a produtividade limitada pela disponibilidade hídrica, $\mathrm{o}$ alto valor do índice de concordância de Willmott $(\mathrm{d}=0,95)$ para o primeiro ciclo de cana-soca indica um bom ajuste dos valores estimados em relação aos valores observados.

\section{Teste do método ZAE}

Uma vez que a calibração e a parametrização mostraram bons resultados, testou-se o método ZAE para a região do Triângulo Mineiro.

A Figura 3 apresenta a variação da curva de produtividade média estimada pelo método ZAE e da produtividade média observada, segundo dados do IBGE, para a região do Triângulo Mineiro. Observa-se que as estimativas de produtividade da cana-de-açúcar pelo método ZAE em função das três épocas de corte, seguiram o comportamento temporal da série histórica de dados médios de produtividade do IBGE. Embora o método ZAE tenha capturado as variações observadas ao longo da série do IBGE, foi constatado um erro sistemático de superestimativa da produtividade.

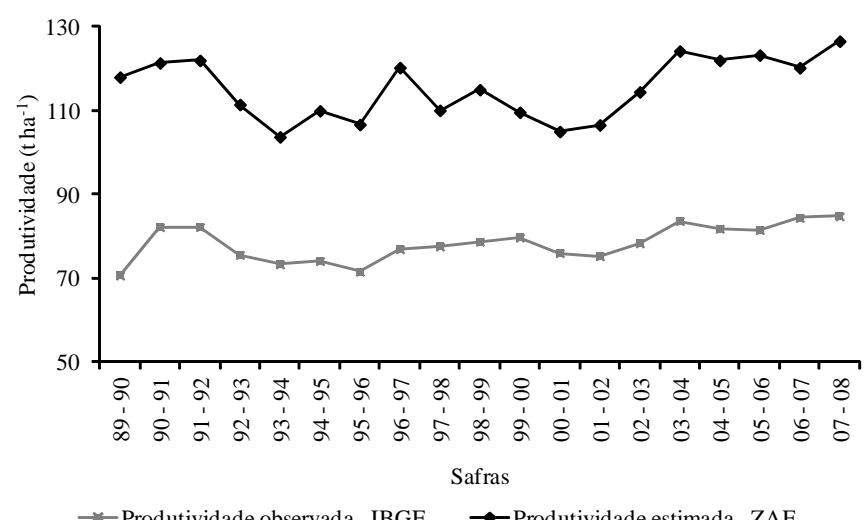

Figura 3. Distribuição temporal da produtividade média estimada pelo método ZAE e da produtividade média da região do Triângulo M ineiro (IBGE)

A Figura 4 apresenta a relação entre a produtividade limitada pela disponibilidade hídrica estimada pelo método ZAE e a produtividade observada da cana-de-açúcar, segundo dados do IBGE, tal como, também, os indicadores estatísticos RMSE, MAE, MBE e índice (d). Nota-se que o método ZAE explica $55 \%$ da variabilidade dos dados médios do IBGE. Verificou-se, pelo teste $t$ de significância dos coeficientes da análise de regressão, que o coeficiente linear da Equação pode ser desprezado em virtude de não ser estatisticamente significativo a nível de 5\% (valor $\mathrm{p}=0,4234$ ).

A análise da Figura 4 indica um erro sistemático por parte do método ZAE $\left(\mathrm{MBE}=37,0 \mathrm{t} \mathrm{ha}^{-1}\right)$, com tendência de superestimativa da produtividade de colmos. A produtividade média estimada também apresenta uma variabilidade maior quando comparada com os dados de produtividade do IBGE $\left(\right.$ RMSE $\left.=37,3 \mathrm{tha}^{-1}\right)$. O baixo valor do índice $d$ indica pouca concordância entre a produtividade média estimada pelo método ZAE e a produtividade observada, segundo dados do IBGE, para a região do Triângulo Mineiro.

Destacam-se os dados de produtividade do IBGE, que correspondem a uma média regional da produtividade dos 


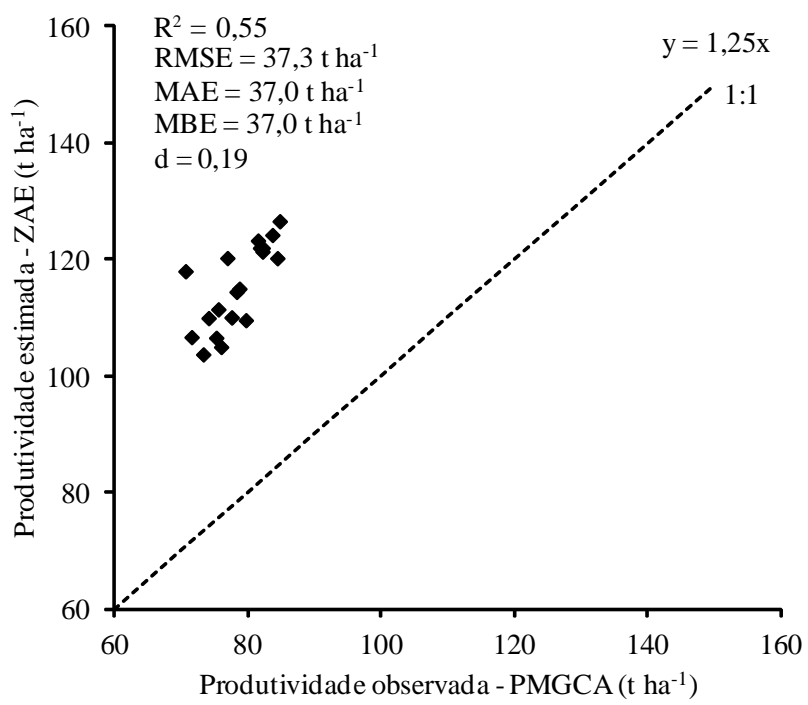

Figura 4. Kelaçao entre a produtıvıdade medıa estımada pelo método ZAE e a produtividade média da região do Triângulo Mineiro (IBGE)

municípios que compõem a região do Triângulo Mineiro, incluindo municípios com alta (compreendendo fazendas com altíssimo nível de tecnologia, manejo e genética) e baixa produtividade (produtores cujo cultivo é exposto a todos os tipos de adversidade). Com isto, a variabilidade dos dados diminui ocorrendo suavização da variância.

Como o MBE representa o erro médio da estimativa e este, por sua vez, apresentou uma superestimativa de $37,0 \mathrm{t} \mathrm{ha}^{-1}$, adotou-se como procedimento de correção a remoção desta tendência média, obtendo-se uma nova distribuição da produtividade média estimada pelo método ZAE para a região do Triângulo Mineiro, conforme apresentado na Figura 5.

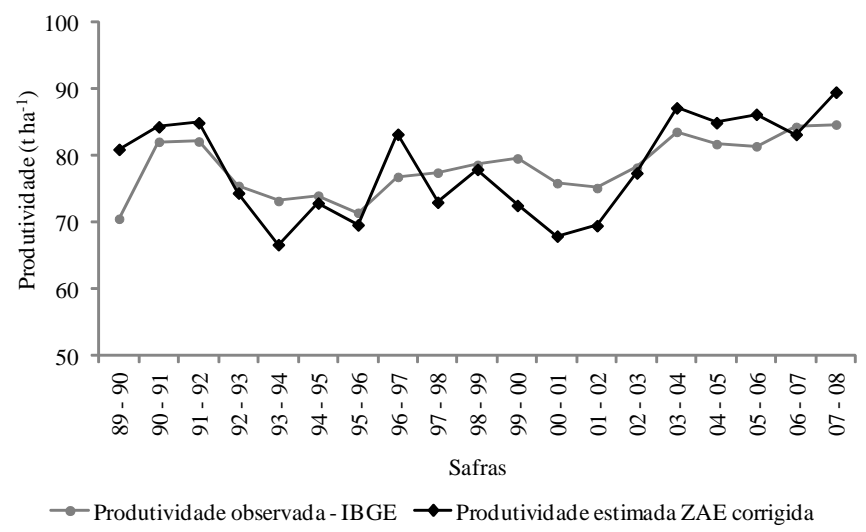

Figura 5. Distribuição temporal das médias corrigidas de produtividades estimadas pelo método ZAE e da produtividade média da região do Triângulo Mineiro (IBGE)

A produtividade média corrigida para região do Triângulo Mineiro acompanha o comportamento temporal da série histórica de dados de produtividade média da cana-de-açúcar segundo dados do IBGE, embora tenha ocorrido, em algumas safras, uma pequena subestimação pelo método ZAE; porém, como os dados representam a média de toda a região e essas subestimativas seguem um padrão de queda também observado na série histórica do IBGE, as estimativas de produtividade são satisfatórias. A Tabela 3 apresenta a produtividade média observada dos dados do IBGE para o período de 1989 a 2008, a média estimada pelo modelo ZAE sem ajuste, a média estimada ajustada e respectivos desvios padrão. Com a correção utilizada (remoção do viés médio, MBE), constata-se que a produtividade média estimada se torna idêntica à produtividade média observada; contudo, a variabilidade na produtividade estimada não é alterada em relação à produtividade sem ajuste, o que é esperado, uma vez que a correção se consistiu em remover a tendência de superestimativa de $37 \mathrm{t} \mathrm{ha}^{-1}$, deslocando para baixo os valores de produtividade estimados em 37 unidades, o que não altera a variabilidade (Tabela 4).

Tabela 4. Valores estatísticos da produtividade

\begin{tabular}{lrc}
\hline \multirow{2}{*}{ Produtividade } & Média & \multicolumn{2}{c}{ Desvio padrão } \\
\cline { 2 - 3 } & \multicolumn{2}{c}{ t ha $^{-1}$} \\
Observada & 78,3 & 4,3 \\
Estimada sem ajuste & 115,3 & 7,3 \\
Estimada ajustada & 78,3 & 7,3 \\
\hline
\end{tabular}

Finalmente, a Figura 6 apresenta a relação entre a produtividade estimada corrigida e a produtividade observada segundo os dados do IBGE e o desempenho estatístico dos erros RMSE, MAE, MBE e índice d para estimativa corrigida de produtividade média para a região do Triângulo Mineiro.

Observa-se o aprimoramento no desempenho estatístico do método ZAE para estimativa de dados médios de produtividade na região do Triângulo Mineiro (Figura 6). Os baixos valores de RMSE e MAE demonstram que a variabilidade detectada antes do ajuste, diminuiu consideravelmente. $\mathrm{O}$ valor do MBE $=0 \mathrm{t} \mathrm{ha}^{-1}$ mostra que, na média e após a correção, o método apresentou-se bem coerente com os dados do IBGE.

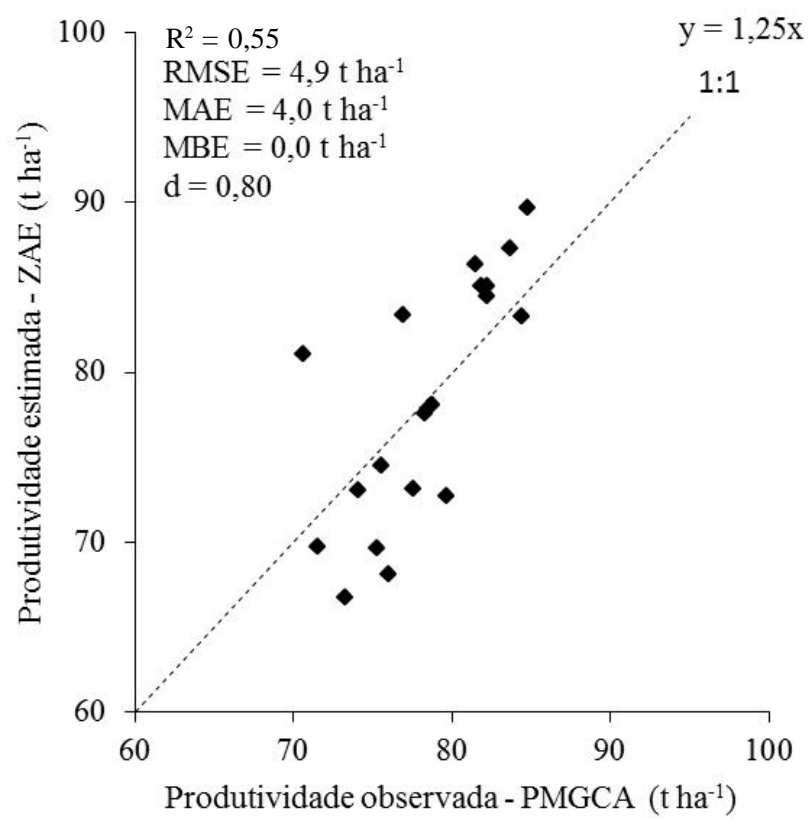

Figura 6. Relação entre produtividade média estimada pelo método ZAE corrigida e a produtividade média da região do Triângulo M ineiro (IBGE) 
Similarmente, o índice $d$, que antes do ajuste era 0,19 , aumentou para 0,80 , indicando boa concordância entre os dados estimados e os observados.

\section{CONClusÕES}

1. A adaptação realizada no método da Zona Agroecológica, permitiu estimar adequadamente os valores de produtividade da cultura da cana-de-açúcar penalizada em função do déficit hídrico relativo, nas principais regiões produtoras do Estado de Minas Gerais, de modo que seja possível verificar os efeitos do clima (época e locais) sobre os valores observados de produtividade.

2. Este modelo se apresentou como importante ferramenta para estimar a produtividade da cana-de-açúcar em macroescala, podendo auxiliar no planejamento da expansão otimizada da atividade no Estado.

3. A validação do método da Zona Agroecológica para a região do Triangulo Mineiro mostrou, após a correção da tendência de superestimativa da produtividade, um bom ajuste em relação aos dados do IGBE, corroborando com a eficácia do método ZAE e mostrando que, desde que sejam feitos os ajustes necessários, o método pode ser aplicado para previsão de produtividade, obtendo-se então resultados satisfatórios.

\section{LITERATURA CITADA}

Allen, R. G.; Pereira, L. S.; Raes, D.; Smith, M. Crop evapotranspiration. Rome: FAO, 1998. 300p. Irrigation and Drainage Paper, 56

Almeida, A. C. S.; Souza, J. L.; Teodoro, I.; Barbosa, G. V. S.; Filho, G. M.; Júnior, R. A. F. Desenvolvimento vegetativo e produção de variedades de cana-de-açúcar em relação à disponibilidade hídrica e unidades térmicas. Ciência e Agrotecnologia, v.32, p.1441-1448, 2008.

Bonnecarrère, R. A. G.; Dourado Neto, D.; Martin, T. N.; Pereira, A. R.; Manfron, P. A. Estimativa das produtividades potencial e deplecionada da cultura de milho no Estado do Rio Grande do Sul em função das condições climáticas. Revista Brasileira de Agrometeorologia, v.15, p.280-288, 2007.

Bristow, K.; Campbell, G. S. On the relationship between incoming solar radiation and daily maximum and minimum temperature. Agricultural and Forest Meteorology, v.31, p.159-166, 1984.

Dantas Neto, J.; Figueirêdo, J. L. C.; Farias, C. H. de A.; Azevedo, H. M. de; Azevedo, C. A. V. de. Resposta da canade-açúcar, primeira soca, a níveis de irrigação e adubação de cobertura. Revista Brasileira de Engenharia Agrícola e Ambiental, v.10, p.283-288, 2006.

Delgado-Rojas, J. S.; Barbieri, V. Modelo agrometeorológico de estimativa da produtividade da cana-de-açúcar. Revista Brasileira de Agrometeorologia, v.7, p.67-73, 1999.

Doorenbos, J.; Kassam, A. H. Yield response to water. Rome: FAO, 1979. 172p. Irrigation and Drainage Paper, 33
Everingham, Y. L.; Smyth, C. W.; Inman-Bamber, N. G. Ensemble data mining approaches to forecast regional sugarcane crop production. Agricultural and Forest Meteorology, v.149, p.689-696, 2009.

Farias, C. H. de A.; Fernandes, P. D.; Azevedo, H. M. de; Dantas Neto, J. Índices de crescimento da cana-de-açúcar irrigada e de sequeiro no Estado da Paraíba. Revista Brasileira de Engenharia Agrícola e Ambiental, v. 12, p.356-362, 2008.

Fischer, G.; Velthuizen, H. V.; Shah, M.; Nachtergaele, F. O. Global agro-ecological assessment for agriculture in the 21st century: Methodology and results. Laxenburg: IIASA, 2002. $155 \mathrm{p}$.

Gilbert, R. A.; Shine Júnior, J. M.; Miller, J. D.; Rainbolt, C. R. The effect genotype, environmental and time of harvest on sugarcane yields in Florida, USA. Field Crops Research, v.95, p.156-170, 2006.

Gouvêa, J. R. F.; Sentelhas, P. C.; Gazzola, S. T.; Santos, M. C. Climate changes and technological advances: Impacts on sugarcane productivity in tropical Southern Brazil. Scientia Agricola, v.66, p.593-605, 2009.

Inman-Bamber, N. G.; Muchow, R. C.; Robertson, M. J. Dry matter partitioning os sugarcane in Australia and South Africa, Field Crops Research, v.76, p.71-84, 2002.

IOMG- Imprensa Oficial de Minas Gerais. <http://www.iof.mg.gov.br/ index.php?/Acao-do-Governo-Arquivo/Producao-de-canade-acucar-bate-novo-recorde.html>. 10 Jun. 2010.

Leal, B. G. Sistema computacional para a avaliação e estimativa das produtividades potencial e real de culturas agrícolas. Viçosa: UFV, 2000. 97p. Tese Doutorado

Liu, D. L.; Bull, T. A. Simulation of biomass and sugar accumulation in sugarcane using a process-based model. Ecological Modelling, v.144, p.181-211, 2001.

Liu, D. L.; Kingston, G.; Bull, T. A. A new technique for determining the thermal parameters of phenological development in sugarcane, including suboptimum and supra-optimum temperature regimes. Agricultural and Forest Meteorology, v.90, p.119-139, 1998.

Marin, F. R.; Lopes-Assad, M. L.; Assad, E. D.; Vian, C. E.; Santos, M.C. Sugarcane crop efficiency in two growing seasons in São Paulo State, Brazil. Pesquisa Agropecuária Brasileira, v.43, p.1449-1455, 2008.

Scarpari, M. S.; Beauclair, E. G. F. Physiological model to estimate the maturity of sugarcane. Scientia Agricola, v.66, p.622-628, 2009.

Silva, L. L.; Costa, R. F.; Campos, J. H. B.; Dantas, R. T. Influência das precipitações na produtividade agrícola no Estado da Paraíba, Revista Brasileira de Engenharia Agrícola e Ambiental, v.13, p.454-461, 2009.

Singels, A.; Donaldson, R. A.; Smit, M. A. Improving biomass production and partitioning in sugarcane: theory and practice. Field Crops Research, v.92, p.291-303, 2005 a.

Singels, A.; Smit, M. A.; Redshaw, K. A.; Donaldson, R. A. The effect of crop start date, crop class and cultivar on sugarcane canopy development and radiation interception. Field Crops Research, v.92, p.249-260, 2005 b. 
Tejera, N. A.; Rodés, R.; Ortega, E.; Campos, R.; Lluch, C. Comparative analysis of physiological characteristics and yield components in sugarcane cultivars, Field Crops Research, v.102, p.64-72, 2007.

Thornthwaite, C. W.; Mather, J. R. The water balance. Publications in Climatology. New Jersey: Drexel Institute of Technology, 1955.104p.
Tubiello, F. N.; Fischer, G. Reducing climate change impacts on agriculture: Global and regional effects of mitigation, 20002080. Technological Forecasting \& Social Change, v.74, p.1030-1056, 2007.

Willmott, C. J. Some comments on the evaluation of model performance. Bulletin of the American Meteorological Society, v.63, p.1309-1313, 1982. 